\title{
Abilities of Village Health Volunteers in Determining Drug Related Problems for Diabetes and Hypertension Patients
}

\author{
Parina Na Pathalung ${ }^{1}$, Phayong Thepaksorn ${ }^{2}$ \\ ${ }^{1}$ Pharmacy Technique Department, Sirindhorn College of Public Health, Trang, Thailand \\ ${ }^{2}$ Trang Research Center for Occupational Health, Sirindhorn College of Public Health, Trang, Thailand
}

\begin{tabular}{l}
\hline Article Info \\
\hline Article history: \\
Received Sep 24, 2017 \\
Revised Nov 20, 2017 \\
Accepted Dec 6, 2017 \\
\hline
\end{tabular}

\section{Keyword:}

Diabetes

Drug use

Health volunteer

Hypertension

\begin{abstract}
The objective of this study was to assess the Village Health Volunteers (VHVs) on their knowledge and skills for determining drug-related problems (DRPs) for hypertension (HTN) and diabetes (DM) patients. This study was conducted among 36 VHVs between March and October, 2014 in ten districts of Trang Province. The semi-structured questionnaire interviews and tests have been developed according to knowledge and skills based health determinants for DRPs. The DRPs training program was assigned including, 1) a short course training (2-day) and hands-on training (1-week) followed up, 2) field work for determining DRPs (16-week) and 3) an assessment for determining DRPs in knowledge and competent skills. All completed data of 25 female VHVs were analyzed with age of 42.93 years old on average. About $68 \%$ were age higher than 40 years old and most of them were Para rubber farmers $(76 \%)$. Most of them had a primary education level $(60 \%)$ and almost a half had more than ten years in VHVs' service $(\mathrm{n}=12 ; 48 \%)$. Their knowledge scores for determining DRPs were 12.88 on average, in total of 15 points. Their overall scores for skills presented were 35.48 out of 50 points in total. In conclusion, this program can enhance VHVs' abilities for determining DRPs for HTN and DM patients. Our findings may contribute to future development of VHVs program for improving VHVs competent skills for DRPs chronic diseases.
\end{abstract}

Copyright $(0) 2017$ Institute of Advanced Engineering and Science. All rights reserved.

\section{Corresponding Author:}

Phayong Thepaksorn,

Trang Research Center for Occupational Health,

Sirindhorn College of Public Health, Trang,

P.O. Box 174 Trang 92000, Thailand.

Email: phayong@scphtrang.ac.th

\section{INTRODUCTION}

In Thailand, Village Health Volunteers (VHVs) are playing key role in health promotion and health prevention for Thai's population incorporated with health officials for more than three decades. VHVs are recruited by the health authorities based on their attributes as community leaders and their willingness to serve as VHVs. They are working closely with populations in community. Apart from health promotion and education regarding maternal and child health, VHVs have been worked successfully in several tasks in communicable diseases elimination and eradication campaigns [1]-[2]. They also participated in drug's use such as non-prescription drugs, drug delivery oral contraceptive for women under supervision of health officials, etc. VHVs are continuously trained for strengthening their roles in different modules by Health Offices of the Ministry of Public Health [3]-[5].

Particularly for the treatment of chronic diseases, hypertension (HTN), diabetes (DM) patients were commonly found to use more drugs than other patients. They are therefore at a higher risk of experiencing drug-related problems (DRPs). DRPs described in the literature include contraindications, drug-drug interactions, adverse drug reactions, prescription errors, and noncompliance with drug use [6]-[7]. DRPs 
following hospital discharge are common among elderly patients using multiple drugs for the treatment of chronic diseases. With respect to older patients with comorbidities and using multiple drugs, DRPs are associated with an increased risk of hospital readmissions, morbidity, and mortality. Discharge of patients from the hospital setting to home care is another important risk factor for DRPs [8].

In Trang study survey, the prevalence of DM and HTN among adults aged more than 35 years old was $1.63 \%$ and $3.69 \%(n=220,066)$ from screening test program in 2013 . This is expected to rise to higher than 5\% in 2020. However, specific population subgroups have a much higher prevalence of the disease than the population as a whole. These subgroups have certain attributes or risk factors that either directly cause diabetes or are associated with it [9]. The correlation of risk factors with development of DM and HTN is never $100 \%$. However, the greater the number of risk factors presents in an individual, the greater the chance of that individual developing or having DM and HNT. Conversely, the chance of an asymptomatic individual without any risk factors having or developing DM and HNT is relatively low [5],[10].

Only few studies have assessed the roles and capacity buildings of VHVs and specific nature of DRPs among DM and HNT chronic patients with multiple drug use discharged from hospital in Thailand [9][11]. In order to achieve the objectives of this study, the authors developed questionnaire assessments and assessed knowledge and skills for determining DRPs for DM and HTN patients in selected areas covering 10 districts of Trang Province. The aim of this study was to assess the VHVs on their knowledge and skills for determining DRPs for HTN and DM patients. Consequently, the suggested tool was included to enhance and improve their abilities to help DM and HTN patients with multiple drug use discharged.

\section{RESEARCH METHOD}

\subsection{Study design and population settings}

As a part of the study on model developing for determining DRPs for DM and HTN patients in Trang Province, this cross-sectional study was conducted between March and October 2014 (8-months) among VHVs living in ten districts in Trang. A sample size of 36 VHVs (3-4 VHVs/ district /Health Center) were recruited and participated in this study. The participants were selected based on stratify samplings according ten districts. Only 25 of 36 participants (70\%) were completing all study procedures and all data employed for analyzes.

The health data online records were accessed and extracted from ten corresponding Community Hospital and Health Centers. In total of 1,000 DM and HTN cases, there were $188 \mathrm{DM}(18.80 \%), 352$ HTN(35.20\%), 120 DM \& HTN(12.00\%), 85 HTN \& Hyperlipidemia(HLM)(8.50\%) and 255 DM, HTN \& Others $(25.50 \%$ ) classified as patients participated and assigned as cases for VHVs in this study (average on age $62.65 \pm 12.90$ years old ; males $=247(24.70 \%)$; females $=753(75.30 \%)$ ). $($ Table I). HTN $(35.20 \%), 120$ DM \& HTN(12.00\%), 85 HTN \& Hyperlipidemia(HLM)(8.50\%) and 255 DM, HTN \& Others $(25.50 \%)$ patients were participated and assigned as cases for VHVs in this study (average on age $62.65 \pm 12.90$ years old ; males $=247(24.70 \%)$; females $=753(75.30 \%)$ ). The report of drug related problems for diabetes and hypertension among patients were present elsewhere.

\subsection{Questionnaire interviews and validations}

The semi-structured questionnaire interviews have been developed according to knowledge and skills based health determinants for DRPs. The assigned program for training DRPs including, 1) short course training for two days and hands-on training followed up for one week in the community, 2) field work practicing for sixteen weeks for determining DRPs in each village, and 3) the evaluation criteria for determining DRPs in knowledge ,skills and competence. Development and validation of the tool have been described elsewhere. A summary of the possible DRPs that could be identified with the checklist is shown in other published study.

There were three categories for questionnaire interviews, including 1) patient chart and historygeneral information for patient, sign and symptom, medication usage and indication, etc. 2) questionnaire interviews for behavioral assessment and 3) questionnaire interviews for determining DRPs. All primary data for patients were conducted by questionnaire interviews and survey at community district hospitals and then trained HVHs were followed up by family visits. The inclusion criteria for case were either HP or DM patients or both to be inconclusive by laboratory test results (FBG>126mg/dl, BP>140/90) [12]-[13]. Using the checklist medication records, hospital discharge prescriptions, interview data, and other relevant information were reviewed by three registered and well-trained community pharmacists. DRPs were categorized according to a number of key items using the Ministry of Public Health classification scheme [12]. 


\subsection{Statistical analysis}

The data analyses were derived by SPSS for Windows (version 18, Chicago, IL, USA). Means and SD were used to characterize including descriptive demographic characteristics, frequencies and percentages. The occurrence of DRPs was calculated by dividing the total number of (potential) DRPs by the number of patients. The number of drugs taken by the patient had a significant effect on the occurrence of DRPs.

\subsection{Ethical aproval}

This study was approved by the ethical committee of Sirindhorn College of Public Health. The permission to conduct the study was granted by Community Hospital Pharmacists and Health Officials from ten districts in Trang Province and participated VHVs. All of the participants were clearly informed of the purpose of this study and agreed by signing a consent form.

\section{RESULTS AND ANALYSIS}

\subsection{Quantitative data}

Twenty-five VHVs were completing all procedure of the program. The mean age of the VHVs was 42.93 years old. More than $68 \%$ were age higher than 40 years old and majority of them were Para rubber farmers $(76 \%)$. They had primary education level $(60 \%)$ and almost an half of them had more than ten years in services $(\mathrm{n}=12 ; 48 \%)$ (Table 1$)$.

Table 1. Descriptive Characteristics of VMVs Who Participated for DRPs

\begin{tabular}{lcc}
\multicolumn{3}{c}{ in Trang Province } \\
\hline \multicolumn{1}{c}{ Characteristics } & No. & Percent \\
\hline Sex & 25 & 100.00 \\
$\quad$ Female & $42.92 \pm 1.31$ & \\
Age (yrs), mean (SD) & $30-59$ & \\
Range(yrs), (min-max) & & \\
Age (yrs) & 8 & 32.00 \\
$\quad<39$ & 11 & 44.00 \\
40-49 & 6 & 24.00 \\
>50 & & \\
Occupation (\%) & 19 & 76.00 \\
Para rubber farmers & 3 & 12.00 \\
Own business/self-employed workers & 3 & 12.00 \\
Temporary workers & & \\
Education level & 15 & 60.00 \\
Primary & 7 & 28.00 \\
Secondary & 3 & 12.00 \\
Associate/college & & \\
Years in services(yrs) & 13 & 52.00 \\
$\quad<10$ & 10 & 40.00 \\
10-20 & 2 & 8.00 \\
$>$ T20 & 25 & 100.00 \\
\hline
\end{tabular}

In this study criteria for evaluation of VHVs knowledge were based on their knowledge for determining DRPs, treatment, food intake, special precaution and recommendations for the patients (total score $=15$ points) whereas skills for determining DRPs were included 1) their participation and involvement for enhancing DRPs for the patients as well as the skills for interviewing on determining DRPs were assessed, 2) testing their knowledge for specifically determining DRPs for DM and HTN, 3) patient outcomes focused were included decreasing a number of cases for DRPs and the quality of life of the patients, 4) they also expected to presented their achievement by presenting and engagement to the audience or assessors and 5) they could provide self-supportive group and their leadership skills for determining DRPs (total score $=10$ points each). VHVs knowledge had 12.88 points on average (total $=15$ points) that presume they had high knowledge for determining DRPs for their assigned patients. This would be correlated with their job experiences for a number of years in services (Table 2). Another part of this study presented classification of DRPs related to medication use, including 1) patient-related problems from non-drug compliance such as non-use every meal, absence of awareness about medication use and thought the symptoms were improved, fearing to side effects, forgotten, fearing to syringes for DM patients, missing appointment with physician, running out of drugs before next visit, etc. 2) side effects of drug use were nausea and vomiting (e.g., metformin), coughing (e.g., enalapril), related side effects of drug use (insomnia, dizziness, blur vision, palpitation, etc. 3) doses too low were using drug sensitivity of degeneration (storage, 
temperature, unfold, etc.) and reducing dose by themselves (thought the symptoms were improved), 4) double medication using by taking drugs over dose (visit different hospital and treatment, physicians) and dose too high from forgotten and then repeating taken drugs.

In this study, the first priority for decreasing DRPs was involved participation and engagement of VHVs interviews for determining DRPs. In addition, the relationships between VHVs and patients were closely since some of them may be a relative or a neighbor who were living in similar community settings. They could provide knowledge and resources as well as communication through their relationships more easily than health officials. Recently, the greater the acceptable of knowledge and skills of VHVs shows capabilities, the greater the enhancing of DM and HTN patients for DRPs [13]-[15]. Second, their knowledge for determining DPRs was a key point for strengthening their roles and implementation. They had skills for determining DRPs for relatively well enough for helping health officials and community pharmacists on DRPs. Even though their education levels were low, they could provide tacit knowledge from their long time experiences. These notices on why VHVs were acceptable for their works and practices [16]. They presented 35.48 out of 50 points in total or 7.09 point on average. Third, this study focused on patient outcomes for decreasing DRPs and improving the quality of life of patients. Fourth, self-supportive group and leadership skills are important for maintaining their work and continuously improving DRPs for chronic patients. This study showed similar results as other criteria skills. However, they were low in presentation on their works by oral presentation that would be correlated with their levels of education [17]-[18].

Table 2. Evaluation Criteria of VHVs Knowledge and Skills for DRPs in Trang Province

\begin{tabular}{llcc}
\hline \multicolumn{1}{c}{ Criteria } & \multicolumn{1}{c}{ Descriptive for evaluation } & Mean & SD \\
\hline Knowledge skills & Knowledge of determining DRPs & 12.88 & 0.18 \\
I. Participation & $\begin{array}{l}\text { Participation and involvement, } \\
\text { skills for interviews for determining DRPs }\end{array}$ & 7.76 & 0.83 \\
TI. Knowledge & $\begin{array}{l}\text { Testing knowledge for determining DRPs } \\
\text { for DM and HTN }\end{array}$ & 7.60 & 0.76 \\
III. Patient outcome & $\begin{array}{l}\text { Decreasing DRPs, quality of life of patients } \\
\text { Oral presentation techniques and }\end{array}$ & 7.72 & 0.61 \\
IV. Presentation & engagement of audiences & 5.88 & 1.16 \\
V. Self-reliance & \multicolumn{1}{c}{ Telf-supportive group and leadership skills } & 7.52 & 0.77 \\
& \multicolumn{1}{c}{ Total } & 35.48 & 2.88 \\
\hline
\end{tabular}

\subsection{Qualitative data}

The descriptive of an example case of VHVs for enhancing DRPs for HTN patient. "When one of VHVs visited HTN female patient, the patient had $65 \mathrm{~kg}$ and her BP were 190/90 $\mathrm{mmHg}$. She liked to eat salty food and rarely exercise. She has often forgotten taking medicine. After she participated in the program by helping of VHVs, she had higher motivated and presented her progress on reducing her high blood pressure. She made her own calendar and notes for taking medications and food intakes in each single day. She reduced consuming food with less salt and increased consuming vegetables. In each day, she sank her feet on herbal warm water after finishing her work. She can reduce her BP to $160 / 59 \mathrm{mmHg}$ within one month for participation program with advice of VHVs".

\subsection{Study limitations}

This study has some limitations. First, only a few of participants were enrolled to participate in this study. It would be better to recruit more number of VHVs for generallazation the results. Second, within the cohort of this study we did not conduct the baseline or follow-up study to confirm consistency of their knowledge and skills. Third, we did not have the comparison data on knowledge for baseline and post VHVs' gaining; thus, clearly this data deserves further evaluation. However, the strengths of this study lie in its representative VHVs from all districts covering subjects and different measured domains of DRPs aspects. The following up and sufficient numbers of cases enable the required statistical analyses to be performed. Also, because of the existing online program access, the data on medical records were reliable and inclusive. To be useful in determine DRPs on risk assessment, tools or questionnaire surveys need to be extensive in their ability to increase patients at risks who living with DM and HTN diseases [19]-[20]. 


\section{CONCLUSION}

Applying knowledge and skills for determining DRPs have several advantages, including a convenient, good relationships, less time consuming and cost-effective way for evaluating knowledge and skills on health risks of DRPs and patient compliance; specifically, this program can apply as a tool for enhancing VHVs' abilities for determining DRPs for HTN and DM patients. Our findings may contribute to future development of VHVs program for improving VHVs competent skills for DRPs chronic diseases.

\section{ACKNOWLEDGEMENTS}

The authors would like to thank all participants, including VHVs, Community Hospital Pharmacists and Health Officials from ten districts in Trang Province for their assistance and participations in this project. This study was funded by the National Health Security Office.

\section{REFERENCES}

[1] Chuengsatiansup K. and P. Suksud," "Potentials and developmental strategies health volunteers in the context of changes," Inst. Social Research \& Health, 2006.

[2] Kowitt D., et al., "Workers as Agents of Health Promotion: Analyzing Thailand's Village Health Volunteer Program," J Com Health, vol. 40, pp. 780-88, 2015.

[3] Phomborphub B., et al., "Village Health Volunteer Participatory in Tuberculosis Control in Southern Thailand," $S$. Asian J Trop Med Pub H, vol/issue: 39(3), pp. 542-48, 2008.

[4] "WHO Regional of South-Ease Asia Office. Role of Village Health Volunteers in Avian Influenza Surveillance in Thailand," 2007.

[5] "Primary Health Care Division. Manual for Assessment Chronic Disease for Village Health Volunteer," 2010.

[6] Stafford A. C., et al., "Drug-related problems identified in medication reviews by Australian pharmacists," Pharm World Sci., vol. 31, pp. 216-223, 2009.

[7] Vinks T. H., et al., "Identification of potential drug-related problems in the elderly: the role of the community pharmacist," Pharm World Sci., vol. 28, pp. 33-38, 2006.

[8] Ahmad A., et al., "Identification of drug-related problems of elderly patients discharged from hospital," Patient Pref Ad., vol. 8, pp. 155-165, 2014.

[9] Trang Provincial Health Office, 2014

[10] Ngousangsai P. S. P. and Puttrabenjapol S., "A Case Study of Health Literacy of Village Health Volunteers," Isan J Pharm Sci., vol. 9, pp. 82-7, 2014.

[11] Komwong D. S. T., "Factors affecting drug use behaviors of village health volunteers," Thai Pharm Health Sci.J., vol/issue: 7(3), pp. 121-6, 2012.

[12] Department of Health Services Support, "Guideline on Health Promotion and Behavioral Risk Reduction of Disease Control for Village Health Volunteers," Ministry of Public Health, 2014.

[13] Teinthavorn V., "Surveillance, Control and Prevention Systyem of DM and HT in Thailand: Policy in Action," Department of Health Services Support. Ministry of Public Health, 2012.

[14] Wanitkun N., et al., "Building equity in chronic disease management in Thailand: A wholesystem provincial trial of systematic, pro-active," Chron Illness, vol. 7, pp. 31-44, 2011.

[15] Sranacharoenpong K. and Hanning R. M., "Diabetes prevention education program for community health care workers in Thailand," J Com Health, vol/issue: 37(3), pp. 610-618, 2012.

[16] Promthet S., et al., "Evaluation of Health Education in the Multi-Professional Intervention and Training for Ongoing Volunteer-based Community Health Programme in the North-East of Thailand," Asian Pacific J Cancer Prev., vol. 13, pp. 1753-55, 2012.

[17] Visanuyothin S., et al., "Health literacy of village health volunteers in Municipalitiy, Nakhon Ratchasima, Thailand," J Pub Health Dev., vol/issue: 13(1), pp. 37-54, 2015.

[18] Hersh L., et al., "Health Literacy in Primary Care Practice," Am Fam Physician, vol/issue: 92(2), pp. 118-24, 2015.

[19] Department of Health Services Support, "Maunal for Community Health Management Leader for Integrated Tambol Management in Health Care," Ministry of Public Health, 2016.

[20] Sriwanichakorn S., et al., "Management system for chronic diseases: a case study of diabetes and hypertension," Health System Research Institute, 2010. 